\title{
MDO and Cross-Disciplinary Practice in R\&D: A Portrait of Principles and Current Practice
}

\author{
Anna-Maria Rivas McGowan ${ }^{1}$ \\ NASA Langley Research Center, Hampton, VA, 23681, USA \\ Panos Y. Papalambros ${ }^{2}$ and Wayne E. Baker ${ }^{3}$ \\ University of Michigan, Ann Arbor, MI, 48109, USA
}

\begin{abstract}
For several decades, Multidisciplinary Design Optimization (MDO) has served an important role in aerospace engineering by incorporating physicsbased disciplinary models into integrated system or sub-system models for use in research, development, $(R \& D)$ and design. This paper examines MDO's role in facilitating the integration of the researchers from different single disciplines during $R \& D$ and early design of large-scale complex engineered systems (LaCES) such as aerospace systems. The findings in this paper are summarized from a larger study on interdisciplinary practices and perspectives that included considerable empirical data from surveys, interviews, and ethnography. The synthesized findings were derived by integrating the data with theories from organization science and engineering. The over-arching finding is that issues related to cognition, organization, and social interrelations mostly dominate interactions across disciplines. Engineering issues, such as the integration of hardware or physics-based models, are not as significant. Correspondingly, the data showed that MDO is not the primary integrator of researchers working across disciplines during $R \& D$ and early design of LaCES. Cognitive focus such as analysis versus design, organizational challenges such as incentives, and social opportunities such as personal networks often drove the human interactive practices among researchers from different disciplines. Facilitation of the inherent confusion, argument, and learning in crossdisciplinary research was identified as one of several needed elements of enabling successful research across disciplines.
\end{abstract}

\section{Introduction}

$\mathrm{D}$ esigning Large-Scale Complex Engineered Systems (LaCES) such as aircraft and submarines requires the input of thousands of engineers and scientists whose work is proximate in neither time nor space. Comprehensive knowledge of the system is dispersed among specialists whose expertise is typically in one system component or discipline. This study examined the interactive work practices among such specialists, seeking to improve engineering practice through a rigorous and theoretical understanding of current practice. This research explored current interdisciplinary practices and perspectives during $\mathrm{R} \& \mathrm{D}$ and early LaCES design and identified why these practices and perspectives prevail and persist. To facilitate a holistic understanding of the research topic, an interdisciplinary perspective informed by engineering practice and theories from organization science and psychology was adopted.

\footnotetext{
${ }^{1}$ Senior Aerospace Engineer and Project Manager, Aeronautics Research Directorate, M/S 254, AIAA Associate Fellow.

${ }^{2}$ Professor, Mechanical Engineering and Integrative Systems and Design, 2250 GGBrown, Ann Arbor, MI 48104, AIAA Member.

${ }^{3}$ Professor, Ross School of Business, Sociology, and Organizational Studies, 701 Tappan Street, Ann Arbor, MI 48109.
} 
The study delves into the interdependence of the engineering disciplines and the associated nonhierarchical interactive practices between researchers (depicted in the dotted lines in Figure 1. Rather than focusing on hierarchical practices (depicted in the solid lines) and connecting mathematical models and hardware, this work focuses instead on human-to-human interactions between disciplines and on the perspectives that drive these interactions, posing the following research questions:

1) What are current practices in and perspectives on interdisciplinary interactions during research and development and early conceptual design of large engineered systems?

2) Why might these practices and perspectives prevail and persist? This paper focuses on findings related to Multidisciplinary Design Optimization (MDO) or Multidisciplinary Design Analysis and Optimization (MDAO) functions and their involvement in crossdisciplinary interactions among researchers of different disciplines in large, dispersed organizations. The term MDO is used in the paper for both functions for brevity. While considerable literature is focused on MDO practice, here we seek to understand how this practice influences the human interactive practices among disciplines. The research questions seek to reveal how these

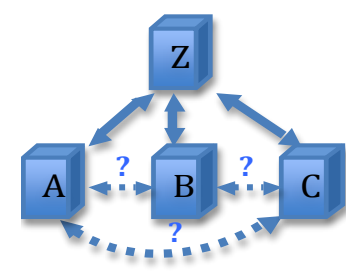

Figure 1 Cross-Disciplinary Interactions interactive connections are made and why, with an overarching goal of understanding how the connections impact subsequent system design. Ultimately, this work seeks to advance the science of designing large engineered systems by improving understanding of some of its precursors during research and development.

This paper begins with background on the context of the research study, followed by an overview of the research methodology. Next, a theoretical analysis differentiating cross-disciplinary research practices is provided. The section on findings that follows provides the synthesized findings related to MDO and includes several examples from the empirical data obtained.

\section{Background}

Several characteristics of the engineered system and the organization that conducts R\&D and early design determined the context of this study. The large size and complexity of the engineered system dictate that the early design effort is not led by a single designer or small group of designers who comprehend the entire system design and can delineate and account for the interdependencies in the work of the relevant engineers and scientists. Rather, "the sheer complexity of many design artifacts means that no one person is capable of keeping the whole design in his/her head and centralized control of the design decisions becomes impractical, so the design process is dominated by concurrent local activities." "From an organizational sensemaking perspective, Weick writes: "Portions of the envisaged system are known to all, but all of it is known to none." 2

Hence, in this study, the focus is not toward a single designer or design group, but rather toward understanding how dispersed researchers interact to enable a potential future system design. While there are several well-utilized system design methods that integrate R\&D results, such as MDO or Quality Function Deployment (QFD), this study focuses on the human-to-human interactions during $R \& D$ and early design that are augmentative to computational or systems engineering methods. Typically for LaCES R\&D and early design, hundreds to thousands are employed at several different geographic locations. The size and geographic dispersion of typical R\&D organizations oblige a focus on organizations and networks of people over a focus on individuals and teams, with an appreciation that the latter creates the former.

Thus, this study examines organization science theories related to connections in organizations, such as distributed and collective cognition, social network analysis, and social capital, some examples of which are in references 3-5. The literature and theories on interdisciplinarity and connections across disciplines provided insight on knowledge integration across domains of study, further described in the next section. The literature from system science, complexity science, system engineering, MDO, and system design also informed the research. Some exemplar references are 6-13. The research design for this study was grounded in qualitative methods. As such, literature on methods in field studies in social psychology and organizations were used, including topics related to surveys, interviewing, observations, ethnography, and grounded theory (references are noted in the next section on research methodology). 
While this study examines interdependencies in engineering and cognition during R\&D in organizations and engineered systems that are both very large, it also examines the related theories in the literature associated with the research topic. Ultimately, this study is a fusion of many different research genres. This fusion is essential for enabling a more holistic analysis of the engineering practices studied.

\section{Research Methodology}

The research questions noted earlier that drove this study were well suited to a qualitative approach as this approach works well for answering "how" questions as opposed to "how many" questions. ${ }^{14}$ The research design consisted of a three-fold, integrative approach that combined survey, interview, and ethnographic research. This approach did not focus on using traditional domain decompositions provided by existing theories. Rather, we focused on inductively finding descriptions based on empirical data from the surveys, interviews, and ethnography. This is a descriptive analysis approach that "attempts to understand cognitive work practices from the perspective of the subject, in the contexts where the subjects find meaning" rather than in a simulated research laboratory environment. ${ }^{15}$ Several references (most of which are noted subsequently) provide considerable information on conducting qualitative research and were used to inform this study. ${ }^{16,17}$ As qualitative analysis is relatively uncommon in engineering, in this paper we provide additional detail on research methodology. Three different research methods were used to help examine different facets of the problem domain. However, the ultimate goal of this study was synthesis of the different data to enable an integrated, rigorous, and comprehensive analysis.

\section{Research Design Strategy}

The overall structure of the research design included a "triangulation" approach where data collected from open-ended surveys, semi-structured interviews, and ethnographic interactions and observations were synthesized. Each research method provided insight into distinct facets of interdisciplinary interactions during engineering systems $R \& D$ and early design. The multi-method approach chosen used:

1) Open-ended surveys to identify current perspectives; ${ }^{18}$

2) Semi-structured interviews to provide detailed, concrete examples of practices; $;^{19}$ and,

3) Insider ethnography to provide a rich, descriptive account of cultural and organizational work life. ${ }^{20-26}$

The research design of the study was guided by principles in qualitative studies. Data were collected using three different methods to allow for synthesis and to strengthen findings during analysis. This approach aided in reducing researcher bias and improving the "trustworthiness" of the findings. Each data collection method unearthed different aspects of interdisciplinary interactions thereby significantly improving the "confirmability" of the findings. Additionally, each of the three data collection methods enabled the opportunity for "negative cases" that challenged preliminary themes. Peer examination from researchers in engineering, organization science, engineering education, and psychology further aided in cross checking interpretations.

Employing insider ethnography also allowed for considerable feedback in the form of sustained member checking from a wide variety of peers within LaCES R\&D. Further discussion on insider ethnography is provided subsequently. Emerson et al. note that "the task of the ethnographer is not to determine 'the truth' but to reveal the multiple truths apparent in others' lives,", 21 for "[any phenomenon] contains multiple truths, each of which will be revealed by a shift in perspective, method, or purpose... The task is not to exhaust the singular meaning of an event but to reveal the multiplicity of meanings, and... it is through the observer's encounter with the event that these meanings emerge" (Mishler, 1970:10, as referenced in 21). Taking advantage of the ethnographic field setting, emerging preliminary findings were presented to members of the organization who were blind to the research questions. Their feedback was integrated and the findings were further refined, and the process was repeated as necessary.

These three methods were integrated into an analytical approach that included first-order analysis of data from each method by itself followed by second-order analysis that integrated data and provisional findings from multiple methods to create updated findings. Then, a comprehensive 
synthesis incorporated relevant theories and created theoretical conceptualizations grounded in the empirical data and backed with theory. Ultimately, such an analytical approach seeks to: 1) present empirical data; 2) explain the data through detailed descriptions; 3) interpret the descriptions through conceptualizations; 4) connect the descriptive and conceptualized findings to the research questions; 5) support the findings with theory; and 6) avoid quantitative framing that can be misleading.

\section{Individual Research Methods}

The survey focused on identifying current perspectives on interdisciplinary interactions by sampling a diverse group of 62 leaders that spanned industry, government, and academia. They provided a unique assessment of current thinking and took place prior to the interviews, which also guided our interview design and analysis. The survey focused on obtaining short, written answers to seven open-ended questions such as: "Please describe things that encourage interdisciplinary interactions" and "Please describe the obstacles to interdisciplinary interaction."

The semi-structured interviews focused on allowing us to obtain detailed, concrete examples of cross-disciplinary practices through the purposeful participant recruitment of 20 practitioners with diverse experiences and responsibilities in aerospace R\&D and conceptual design. The 20 respondents were carefully chosen to provide a balanced sample considering years of experience, job site locations, leadership and staff positions, and diversity of engineering tasks. The interviews offered comparative data "for understanding the world from the view of those studied" and helped to "unfold the meaning of their experiences." "14,19 Example questions asked during these interviews included: "I'm interested in hearing about an experience you had in working with someone outside of (their home area of work). Tell me about it." "Can you describe what challenges you faced?" "I'd like to hear about what you gained from the experience?"

Interactional and observational data collected through an insider ethnographic approach provided a rich, descriptive account of the cultural and organizational work life of R\&D engineers in the aerospace industry. ${ }^{22}$ Ethnographic research for this study was primarily conducted in aerospace R\&D settings via 20 years of insider involvement and extensive interaction with a wide variety of aerospace R\&D and design entities. The long duration of the insider ethnography provided critical insight to discern "the more subtle, implicit underlying assumptions that are not often readily accessible through observation or interview methods alone."21

\section{Integrative Data Analysis Approach: From Codes to Synthesized Analysis}

The overarching analysis approach was interpretive involving qualitative content analysis using theoretical sampling and methods of constant comparison (in keeping with the grounded theory methodology developed by Glaser and Straus). ${ }^{27}$ Data analysis was inductive, guided by constant comparison methods, in which themes were identified, continuously compared to newly emergent themes, and revised based on the comparison. ${ }^{27}$ As is common in a qualitative study, data from all research methods were integrated and re-coded as new findings emerged and the research design was adjusted accordingly. ${ }^{28}$ While a highly inductive data analysis approach guided the findings, to prevent assiduous theory avoidance, this work has theoretical underpinnings in several genres of literature as presented in the previous section. ${ }^{14,29,30}$ Data were coded and re-coded via an iterative first-order and second-order analysis approach.

First-order analysis involved primarily focusing on data from an individual method. Here patterns in the data were examined to identify empirical groupings and descriptions. First-order analysis provided an organized, descriptive account of codes and preliminary themes. After first-order analysis, deeper (second-order) analysis was conducted by integrating the data and preliminary themes from all three methods to provide more dense descriptions of emergent concepts and where possible, also provide explanatory frameworks or conceptualizations to further clarify some phenomena. ${ }^{20}$ For a few major themes, potential explanatory perspectives from existing social science theories were also considered.

In sum, this second-order analysis focused toward providing theoretical perspectives that seek to interpret and explain the first-order analysis. ${ }^{20}$ This "theoretical perspective is grounded in, and emerges from the first-hand data (cf. Glaser and Strauss, 1967)." ${ }^{11}$ Second-order analysis entailed discovering meta-themes that encompassed multiple codes from open-ended survey questions and interview responses and ethnographic observations to derive explanatory conceptualizations to provide a more theoretical perspective on the findings. 
While the goal of the explanatory conceptualizations created in this study is not focused toward building theory, a theory-building lens was used in creating them. "Two foci in developing theory are discovering patterns and identifying processes." 32 Theory may be viewed as "plausible relationships proposed among concepts and sets of concepts." (Strauss and Corbin, '94 as quoted by 32) Thus, our goal in the conceptualizations derived was to understand the "orderly relationships among disparate phenomena." (Goodenough, 1964 as quoted by 20)

The synthesized analysis of the study data was driven by exploring patterns, processes, and relationships and sought to illuminate the actual engineering and scientific practices and perspectives on interdisciplinary interactions. Except where noted, the findings herein are a second-order synthesis of all of the codes, themes, and meta-themes from all data. Triangulation was an essential aspect of the research design.

To echo what is well documented in qualitative research theory literature, we note that a quantitative frame for analysis of the data is an inappropriate frame given the sample size and research methodology used. Accordingly, statistical generalizability is not the aim for this study but rather generalizability in the context of R\&D in LaCES is the appropriate frame for considering potential transferability of these findings to other contexts.

\section{Synthesized Findings from all Three Methods}

As noted earlier, data from all three research methods were integrated to create the synthesized findings presented below. Though specific individuals served as respondents for the survey and interview portion of this research (62 from the open-ended surveys and 20 for the semi-structured interviews), their data were integrated with scores of respondents who provided input for the ethnographic portion of this research. The unit of analysis of this study is a group level of analysis. The synthesized findings represent a triangulated analysis based on input from all sources of data. In presenting the synthesized findings in this paper, supporting data from a wide range of respondents are used with bulleted lists used to indicate answers from different respondents. Where possible, the examples of supporting data for each finding is selected from one or more respondents who articulated the finding most succinctly. However, note that each finding is supported by considerable data from a wide variety of respondents from the different research methods.

\section{Differentiating Cross-Discipline Practice}

In common practice, the various terms used to describe cross-disciplinary work are often interchanged. For example, colloquially, when the MDO community talks of "MDO", we do not limit our discussions to purely "multidisciplinary" research but rather we are typically referring to a broader spectrum of research that is more accurately described theoretically as "cross-disciplinary." In this section, literature on cross-disciplinary research is reviewed to clarify key terms that will be used in this paper.

While definitions of "working across disciplines" are equivocal in many research articles in aerospace literature, literature that focuses on interdisciplinary research is more concise and theoretically consistent. The following definitions are derived based upon several references. ${ }^{33-45}$ Klein's caution on any taxonomy is also warranted: "Taxonomies construct the ways in which we organize knowledge and education. However, they are neither permanent nor complete and their boundaries change." 45

Cross-disciplinarity: Used to indicate all types of interactions between disciplines in this paper.

Multidisciplinarity: The root prefix "multi," means "much or many.",46 In a most rudimentary sense multidisciplinary simply implies the inclusion of multiple disciplines. In a more active sense, multidisciplinarity refers to the combination of multiple disciplines (which may be non-integrative), where each discipline preserves its methodologies and assumptions without significant modification from other disciplines.

Additional insights from the literature are also provided. Based upon the definition developed by the Organization for Economic Cooperation and Development (OECD), Klein defines multidisciplinarity as "an approach that juxtaposes disciplines. Juxtaposition fosters wider knowledge,

5

American Institute of Aeronautics and Astronautics 
information, and methods. Yet, disciplines remain separate, disciplinary elements retain their original identity, any existing structure of knowledge is not questioned. ${ }^{, 45}$ For example, in a multidisciplinary context, different disciplines can be taken into account without active cooperation from the different disciplines. Interestingly, one researcher cites an illustrative example of this as "the engineering profession's effort to include social contexts of practice." ${ }^{45}$

Klein also describes an example of multidisciplinarity as when results of different disciplines are integrated into a common framework. This is a common practice in engineering in some forms of MDO and systems engineering. Repko notes that: "multidisciplinary approaches tend to be dominated by the method and theory preferred by the home discipline. ${ }^{34}$ Quoting a definition provided by the National Academies, Repko describes multidisciplinary research as involving "more than a single discipline in which each discipline makes a separate contribution. ${ }^{34}$

While multidisciplinarity and interdisciplinarity both seek to overcome disciplinary monism, they approach this goal by different means. ${ }^{34}$ Multidisciplinarity is distinguished from interdisciplinarity to account for the relationship between the disciplines. In a multidisciplinary scenario, the relationship between disciplines "may be mutual and cumulative but not interactive." 44 Klein writes: "when integration and interaction become proactive, the line between multidisciplinarity and interdisciplinarity is crossed." ${ }^{45}$ In an interdisciplinary relationship, the practices and conventions of each discipline are interactively blended.

Interdisciplinarity: The root prefix "inter," means "between." fusing and integrating of several disciplines, where each discipline's methodologies or assumptions are interdependent on other disciplines. In interdisciplinarity, the practices and conventions of each discipline are interactively blended such that the disciplines (and disciplinary knowledge) are changed during the integrative process.

Repko notes "interdisciplinarity studies a complex problem (including mega ones) by drawing on disciplinary insights (and sometimes stakeholders views) and integrating them. By employing a research process that subsumes the methods of the relevant disciplines, interdisciplinary work does not privilege any particular disciplinary method or theory." Rather, interdisciplinary research is usually undertaken to advance knowledge that lies beyond any one discipline, yet can still be very focused. "Understood as knowledge integration, interdisciplinarity is not the opposite of specialization. Research can be specialized (i.e., focused on a narrow topic) either within a disciplinary framework or drawing on various disciplines." ([Rafols \& Meyer, forthcoming] from 33) (emphasis added)

Perhaps the most salient discriminator for interdisciplinarity (as compared to multidisciplinarity) is that the individual disciplines and disciplinary knowledge are transformed during the integrative process and are no longer individually and distinctly distinguishable. For example, Klein notes "individuals may find their original disciplinary methods and theoretical concepts modified as a result of cooperation, fostering new conceptual categories and methodological unification (Boden 1999, pp. 19-22). ${ }^{, 45}$ At the greatest level of interdisciplinarity, the core issues and questions of a complex problem may "lack a compelling disciplinary basis, and a critique of disciplinary understanding is often implied. (Lattuca 2001, p. 117),"45

In multidisciplinarity, many different engineering components or disciplinary experts may be brought together to create a new, engineered system by comprehensively including all factors from the individual components derived by the disciplinary practitioners. Yet, the individual hardware or software components (and the corresponding knowledge of the practitioners) are still modular in the integrated system. In interdisciplinary practice, many different disciplinary knowledge bases are proactively blended together to create a new, engineered system by an iterative and reciprocal interplay between disciplinary practitioners - changing disciplinary thinking (and the related engineering components) in the process. The system developed by multidisciplinary means likely will be very different from the system developed by interdisciplinary means.

For example, consider two types of aircraft configurations shown in Figure 2. In a very general sense, some aspects of the major components of an airplane that is a conventional "tube with wings" could be developed through multidisciplinary approaches because major components such as wing, propulsion system, and fuselage are physically connected and certainly coupled, but in a manner that allows the wing, propulsion system, and fuselage to be developed somewhat separately, yet with consideration for connections and coupling. The different components remain distinct in the final system. In a general sense, a hypersonic air vehicle has a wing, propulsion system, and fuselage that 
are so physically enmeshed and coupled that much of the development of this type of vehicle will likely require more interdisciplinary approaches.

Transdisciplinarity: The root "trans," means to go across or beyond. ${ }^{46}$ Transdisciplinarity describes crossdisciplinary scenarios when, during the integrative process, new disciplines emerge and transcend the constructs of existing disciplines. In this process, new practices and conventions are interactively created that transcend the practices and conventions of the original disciplines.

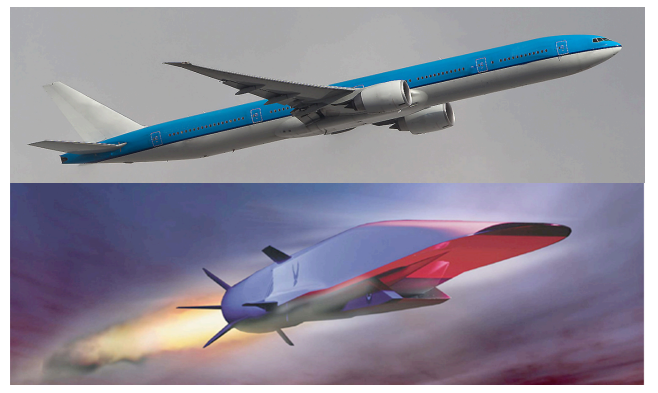

Figure 2 Example Large-Scale Systems

Examples of the above terms in engineering are:

-Multidisciplinarity: Combining a separately developed structural model with an aerodynamic model being mindful of boundary conditions, etc. Operationally, the engineering practitioners in structures and aerodynamics may add the models from the other discipline; however, their cognitive breadth largely remains in their original discipline.

-Interdisciplinarity: Aeroelasticity, which is a study where structural models and aerodynamic models are interactively developed such that each model is a dynamic function of the other. A catastrophic coalescence of the two models is known as flutter. Operationally, practitioners in aeroelasticity have integrated their math models as well as their mental models such that their cognition has changed.

-Transdiscipline: Creating an energy harvesting flutter concept. This concept transcends the interdisciplinary development of modeling the catastrophic event of flutter to exploiting the predictable, nonlinear event for energy harvesting purposes. Operationally, human learning is a quintessential transdiscipline effort for we combine disparate concepts and take them to a new state that may be inspired by but far removed from the original concepts.

-Cross-discipline: All of the above.

\section{Findings}

As noted earlier, this paper draws from the findings of a larger study. We focus attention on several of the findings that relate to MDO's involvement in cross-disciplinary interactions. We begin with a discussion of the benefits of MDO in enabling cross-disciplinary research based upon the significant empirical data obtained in the study. However, an overarching and surprising finding from the study was that while MDO has an important role in enabling cross-disciplinary research, MDO practitioners do not play a significant role in facilitating cross-disciplinary interactions between disciplinary practitioners. Rather, respondents overwhelming noted that cognitive, social, and organizational aspects dominate the interactive arena between disciplines far more so than traditional engineering or mechanical aspects such as concerns of inconsistent meshes in mathematical models nor did the interactions between disciplinary interactions typically rely upon an MDO integrator. To understand these findings with greater depth, we will first note how MDO plays a role in fostering crossdisciplinary research based upon the empirical data received. Then we will examine specific scenarios when MDO was the designated integrator between disciplines. We will conclude with a summary of a few principal challenges in MDO's involvement with cross-disciplinary interactions.

\section{1) MDO's Role In Fostering Cross-Disciplinary Research}

MDO is extremely effective for addressing many integration needs in an organization such as quantifying the system-level trades of different technologies to guide the direction of disciplinary research efforts and to assist managers in developing a technology portfolio. In many organizations, MDO groups often have the stated purpose of integrating input from multiple disciplines in their organizations to evaluate or design macro-system concepts. With consistency, all respondents in this study respected the work of the MDO groups in their organizations stating that they were essential for understanding some systems-level trades. Respondents stated that the systems-level analyses conducted by the MDO groups helps increase awareness of the impacts of disciplines outside a 
researcher's specialty area and is helpful for assisting program managers in determining the allocation of resources for different technologies.

While most respondents noted that MDO did not typically initiate cross-disciplinary interactions, many respondents stated that MDO was sometimes helpful in analyzing the sensitivities of different technologies within a cross-disciplinary effort. A single-discipline researcher with 30 years experience conveyed that MDO: "helps because when I understand what about the other person's activity or discipline is important especially from the global perspective. Then I can better appreciate it and I can try to understand when I should try to work with them a bit more, help them a bit more, or else to just understand the whole problem in general. That sometimes spawns a new idea, actually."

The data showed that developing an enhanced understanding and respect for the importance of other disciplines were key enablers to improved interdisciplinary interactions. Correspondingly, a lack of such interdisciplinary understanding and respect created significant challenges to interactions between disciplines. The educational role of MDO in fostering an improved and mathematically rigorous understanding of different disciplines is a critical role in enhancing cross-disciplinary interactions. However, this role may be over-looked or insufficiently utilized as disagreements regarding interfaces, interactions and interdependencies among disciplines, or how best to use them, was an oft-noted lament of many respondents. An example from one respondent (a senior research leader with over 25 years experience):

"The [discipline A] folks basically said just give us [this interface]. We don't care what you [do in your discipline]. We don't care how much [you do this]. Just make it so it [meets the interface requirements we have]. So, based on that interchange, my general feeling was they felt like they didn't need us. They were dictating the [the interface] and as long as I [did that] they didn't care about what I did in my discipline. [The systems] are very interconnected and no piece works in isolation from the other piece. So, they are all interconnected and they're all trading off. So the reality is that ...I can [meet their interface demands perfectly], no kidding, ... but you're never going to get it [the overall system to work]. And., that's not going to suit their needs. So, the tradeoffs that I have to make in [disciplines B and C] are going to influence [discipline A]. So, if I [just meet their interface condition] we're going to have a non-optimum [system] solution."

This vignette (that captures many sentiments from other respondents) shows that a disregard for the significance of another discipline can create rifts organizationally and dishearten some from proposing future work with another discipline that does not respect the impact of their work. Lack of mutual regard for the technical contributions of another discipline often elevated a technical disagreement to a social disengagement between organizational units. When these disagreements persisted, negative emotions of frustration and anger often followed.

In an opposite scenario, the following respondent (a senior research leader with over 35 years experience) spoke with a sense of validation when another discipline realized that a previously held boundary between them was preventing the other discipline from reaching a challenging engineering goal:

"The [discipline A] guys realized that they cannot build [high performing discipline A sub-systems] to meet the challenging goals without running into [a problem our discipline can fix]. They run into [this problem]; in order to have [their sub-system] perform, you have to have some [work from our discipline]. One [discipline A] guy within the [discipline A line organization] realized that [our discipline B] was important, and then he worked with our [discipline B] guys to say, hey, they feel like you're an important part of [the discipline A subsystem] development. There's a mutual understanding that they need us, and then we can help them. They provide us the knowledge of [their sub-system]. The [discipline A] guy and [our discipline B] guys are co-lead. We don't know much about [discipline A] stuff, so he provides us knowledge about [discipline A]. We do the [discipline B], but then we work together. That's how we do one and one and make it more than two, us working together. It's not like, here is a [discipline A] model; go do the [discipline B] work. We're working together. We're defining the requirements together. He's working with us on developing [a combined test], so it's a collaborative effort. Everybody is seen as a so-called equal partner. Every contribution is valued."

The above two examples, which represent the comments of many respondents, highlight some of the significant social aspects regarding connections between disciplines. The degree to which

8

American Institute of Aeronautics and Astronautics 
disciplines interacted and the quality of their interactions often heavily corresponded to the respect between the groups and the value one discipline placed on the other. The importance of mutuality and reciprocity in working across disciplines was a common theme in this study. This finding ties well with research on building positive social capital in organizations where mutual regard, respect, and reciprocity are a few of the principal tenets. ${ }^{5,47}$ Most respondents in this study described these essential ingredients when discussing what was important in working across disciplines. For many respondents, these social ingredients were more essential than engineering aspects.

The above findings, describe the essential role of MDO in fostering some of the aspects of interactions between disciplines in a large, dispersed engineering organization. MDO can provide strategic technical guidance for researchers and managers; enables the development of systems-level designs by integrating physics-based models from multiple disciplines; educates single-discipline researchers regarding the significance of other disciplines; and several other important roles that are outlined extensively in other literature. ${ }^{6}$ Despite this, the preponderance of the data consistently showed that MDO is not the primary integrator between researchers of different disciplines in everyday $R \& D$ practice, or said differently, MDO is not the primary facilitator of researcher-toresearcher cross-disciplinary interactions in a large, dispersed organization.

This finding was consistently and strongly revealed in the surveys, interviews, and ethnography. In the surveys, although there were many respondents who were MDO practitioners, and MDO exists in nearly all of the organizations represented, there was nearly a complete absence of survey responses related to MDO. Rather, the responses related to social science aspects exceeded the responses related to engineering or mechanical aspects by an extremely wide margin. In sum, respondents clearly identified that cognitive, social, and organizational aspects were the primary influential agents in crossdisciplinary interactions. A comprehensive summary of all of the findings can be found in reference 48. Here, we will focus on elaborating upon a few specific examples of some challenges of crossdisciplinary interactions as they relate to MDO. These examples were chosen based upon their predominance in the data. Our focus here is to better understand current practice to lay the groundwork for future changes to practice.

\section{2) Cognitive, Social, and Organizational Aspects when MDO Serves as the Designated Focal Point of Interaction}

Many respondents described scenarios where a single discipline becomes the system of interest (a micro-system) and the focal point for integrating disciplines. This common practice may be referred to as technology integration and advancement, or more colloquially as technology push. It involves a largely single-discipline team methodically advancing or maturing a technology that was researched and developed primarily within their single discipline area. The technology is advanced or matured by drawing upon a diverse set of other disciplines to support the single-discipline team in analyzing and testing their technology (such as in wind-tunnel testing a specific technology). As described further below, this approach where a single-discipline is a micro-system creates a very different interactive scenario than when a diverse set of disciplines interacts to enable a macro system that is beyond any one discipline. Socially and organizationally, the diverse set of disciplines interacts in a supportive role to the single-discipline that is serving as the integrator. When MDO serves as the focal point of integration, a similar social and organizational scenario takes place.

Respondents noted that the quality of the relationships in the cross-disciplinary interaction varies greatly. When the relationships were positive, long-term social connections can form creating a network of colleagues across a large organization that may build positive social capital. ${ }^{49}$ Though one discipline (or MDO) is the focus of attention and leads the effort, this lead discipline sometimes attends to the needs of the supporting disciplines through seeking "mutual exchange, aid and benefit" among the team members. ${ }^{50}$ In highly reciprocal relationships such as these, the team effort often transcends the original focus in the single discipline/MDO to a number of highly integrated research tasks across different disciplines.

Respondents also noted that when the quality of the relationships between the primary single discipline/MDO and supporting disciplines is professional but less reciprocal, interactions can degrade to an approach centered upon simply meeting the stated requirements of the single discipline/MDO with brevity. A team leader of a single-discipline focused effort who has over 30 years experience provides an example: "Any good cross-disciplinary work I've done I've found that the other person has to develop some interest in your problem. Otherwise they're just a turn-key to deliver a product 
and then you're never going to quite engage enough to where they really can deliver fully what you need. They'll just do the minimum. Whatever you wrote down in your requirements document, 'here it is goodbye.",

This was a frequent lament of many respondents regarding working with the MDO groups in their organization. Many respondents viewed their interactions with the MDO group as working in a supporting role to MDO rather than in collaboration with the MDO group to further the evaluation or design of a macro-system concept of interest. Thus, most respondents saw the MDO group as a type of chief connector or coordinator to whom they supplied input. Few respondents viewed the MDO group as fellow collaborators and only a very small number of respondents indicated that they worked with the MDO group in a collective manner. One MDO researcher with over 30 years experience replied that: "we do integrate from different disciplines into our model, but we do not require them to integrate."

It is possible that the degree of reciprocity in the relationship between MDO and the supporting disciplines is a key factor. While all respondents highly valued the macro-system level, integrative work the MDO groups accomplished, respondents who worked with the MDO groups in a more reciprocal relationship were significantly more positive about their interactions with MDO. In these scenarios, the MDO researchers proactively sought more collaborative and collective interactions because they "think that kind of flow of information and ideas is important," (from an MDO researcher with 10 years experience). Another MDO researcher with 30 years experience described, "You sit down at the table and you're sort of figuring out what's this person requiring of me or needing of me? Can I help them? Can I elicit from them what I need? Vice versa, can I give them something in return, sort of an- almost trading?"

Respondents who noted little reciprocal benefit to working with the MDO group described their interactions more like paying a needed organizational tax than working in a mutually beneficial team arrangement. They described their supportive role to MDO as closer to a transaction of information. In recounting their time on an MDO-led team, a single-discipline researcher with 20 years experience states that they "never got anything out of it." Correspondingly, some MDO researchers described their interactions with other disciplines as largely a one-way interaction. An MDO researcher with 30 years experience describes the following:

"Mainly we need input from all the different disciplines. We probably can give back a little bit of information, but it's mostly them feeding us, I guess. ... The MDAO [group] is really the [group] that has to do all the connecting. We are the cross-disciplinary, multidisciplinary group, so we have to do that connection. I think it's mostly a onedirectional type thing. Which maybe is part of the problem as far as why they don't like working with-sometimes don't jump at working with us is because they have to give information to us and we're not providing much back to them, and so they feel like it's more of a chore for them ... when we're not giving them information back."

Another MDO researcher noted that when their group is working on a conceptual design of a macro-system, many of the supporting disciplines do not always feel like they are part of the macro system conceptual design team, although the macro-system concept is dependent upon the input of the supporting disciplines.

For all scenarios where a single-discipline or group (such as MDO) was the focal point of the cross-disciplinary interaction, the social relationship between the lead discipline or group and the supporting disciplines was paramount. In contrast to a scenario where all disciplines are working together to create a macro-system, having a single discipline or group as the focus of integration creates an implicit social or organizational hierarchy among disciplines that, in reality, are equivalent peers. The degree of mutuality or reciprocity in the interactions was a consistent factor in the ultimate quality of the cross-discipline efforts.

\section{3) The Different Foci of Single-Discipline and MDO Research}

Regarding evaluating unconventional concepts, several single-discipline researchers stated that MDO was less effective in this area. One of the chief challenges in working with unconventional concepts with MDO that respondents (both MDO researchers and single discipline researchers) stated is a mismatch in fidelity between single-discipline researchers and MDO researchers and systems analysts. Where MDO and system analysts need low fidelity models in order to efficiently evaluate a system, yet the single-disciplinary researchers are working toward higher fidelity in terms of modelling 
and understanding the new technology. The challenges here are multifaceted, frustrating both MDO researchers and single-discipline researchers. A few of these challenges are summarized below.

\section{Fidelity of physics-based models:}

Lower fidelity models are not of interest to many single-discipline researchers as many view them as a step backwards in technology maturation. This creates challenges with career advancement and peer respect in the single disciplines and it stymies progress in MDO. The lower fidelity information needed by the systems analysts may be based on parameters from conventional concepts, which may not capture the principal benefits of the new technology developed by the single-discipline researcher, which discourages the single-discipline researcher who would like to more fully use the capabilities of their new concept. However, the single-discipline researcher may not fully know how to create a model that is appropriate for MDO research.

\section{Incentive system:}

Based on the overwhelming response from the interviews and ethnography, the incentive system for research through early conceptual design work appears to be focused on individual achievement, largely measured in the number of papers published. Responses regarding the incentive system were frequently volunteered and pointed. Many suggested that the most significant challenge is clearly identifying what an individual has done on an integrated effort. In Table 1, line managers and researchers describe the emphasis on individual accomplishment.

\section{Table 1 Descriptions Regarding the Individuality Emphasized in the Incentive System}

\section{Line managers (all supervisors) remarked:}

- "That's the hard part, because our mindset is that everyone has to do their fair share, and I have to have a clear way of measuring and documenting a person's contributions. It is hard to identify what someone's done, but I think that's the role of the local supervisor."

- "You're still graded on your own individual performance. That's the whole system- is grading on individual performance, not how you contributed to a team becoming better. ... [the promotion process for research] encourages people to pursue their own individual thing as opposed to working in a team,"

- "I think we still as an organization reward someone who's narrow and deep - as a researcher, not as a Project Manager, but as a researcher."

The researchers interviewed echoed these sentiments, often describing the need to "get credit." All three researchers with greater than 20 years experience remarked:

- "One of the blocks to collaboration is-there are a lot of obvious ones, but egos are a big thing, and who's going to get credit for something. Those aspects can stand in the way, and getting credit for an idea affects people's career advancement."

- "They're afraid that if they share what they're working on, someone is going to steal credit for their work."

- "If I work on this, it might be a dead end and I don't get a reward, and it's very difficult for me to go through the [line manager's] evaluation process: 'What great things I did for the project? Oh, I just worked with this guy and did some studies, 'and who wants a study?"

As noted above, during R\&D in many LaCES organizations there is not significant incentive for the single discipline researcher to take the effort to create a model for interdisciplinary design.

\section{Cognitive and work focus:}

Cognitively, the MDO researchers and single-discipline researchers are often focused toward different directions. Whereas MDO researchers mostly work towards design, many single-discipline researchers mostly work towards analysis. Table $\mathbf{2}$ is a high-level contrastive summary that provides a descriptive account of the two prevailing approaches to work that emerged from the study, recognizing that the boundaries between the two approaches are indistinct. Hence, this summary should not be viewed as either exhaustive or exclusive. Table 2 derived from an analysis of the culture in the organization regarding working across disciplines. While definitions of organization culture vary in the literature, several key constructs are common among researchers. These constructs include values, 
assumptions, and behavioral norms that define the way in which an organization conducts its business. $^{51-53}$

Table 2: Summary of Two Interdependent Approaches (Source: the Author)

\begin{tabular}{|c|c|c|}
\hline & $\begin{array}{l}\text { Physics-Oriented, Single-Discipline- } \\
\text { Focused Research }\end{array}$ & $\begin{array}{l}\text { Design-Oriented, Capability-Focused } \\
\text { Research }\end{array}$ \\
\hline \multirow{2}{*}{$\begin{array}{l}\text { Values: } \\
\text { Enduring } \\
\text { Beliefs }\end{array}$} & Focus is within a discipline & $\begin{array}{l}\text { Focus is on using discipline knowledge for } \\
\text { a concept that requires multiple disciplines }\end{array}$ \\
\hline & $\begin{array}{l}\text { Deep understanding and analysis of } \\
\text { phenomena within a discipline }\end{array}$ & $\begin{array}{l}\text { Creating a new system capability which is } \\
\text { sometimes focused on solving a system } \\
\text { problem }\end{array}$ \\
\hline \multirow{3}{*}{$\begin{array}{l}\text { Values: } \\
\text { Shared } \\
\text { Symbolic } \\
\text { Systems for } \\
\text { Decision } \\
\text { Making } \\
\text { Criteria }\end{array}$} & Technical papers & $\begin{array}{l}\text { Difficulty, novelty, or potential benefit of } \\
\text { the new capability or problem solved }\end{array}$ \\
\hline & $\begin{array}{l}\text { Demonstrated level of understanding } \\
\text { of the physics of a phenomenon }\end{array}$ & $\begin{array}{l}\text { Demonstration of the capability or solved } \\
\text { problem }\end{array}$ \\
\hline & $\begin{array}{l}\text { External recognition within one's } \\
\text { technical area/ discipline }\end{array}$ & External recognition by potential users \\
\hline \multirow[b]{2}{*}{$\underline{\text { Assumptions }}$} & $\begin{array}{l}\text { Among the international leaders in } \\
\text { technical understanding within a } \\
\text { discipline area }\end{array}$ & $\begin{array}{l}\text { Among the international leaders in system } \\
\text { or subsystem capability }\end{array}$ \\
\hline & $\begin{array}{l}\text { The journey toward understanding } \\
\text { and analysis is beneficial regardless of } \\
\text { the ultimate research outcome }\end{array}$ & $\begin{array}{l}\text { The journey toward developing the new } \\
\text { capability or solving the problem is } \\
\text { beneficial regardless of the ultimate } \\
\text { research outcome }\end{array}$ \\
\hline \multirow[t]{2}{*}{$\frac{\text { Behavioral }}{\underline{\text { Norms }}}$} & $\begin{array}{l}\text { Whether working internally or } \\
\text { externally, conduct the work } \\
\text { individually or in small groups largely } \\
\text { within a single discipline }\end{array}$ & $\begin{array}{l}\text { Work with the requisite disciplines } \\
\text { internally or externally to enable a viable } \\
\text { system, sub-system, technology or } \\
\text { capability or problem resolution }\end{array}$ \\
\hline & $\begin{array}{l}\text { Seeking improvements within the } \\
\text { discipline }\end{array}$ & $\begin{array}{l}\text { Seeking improvements for a system, sub- } \\
\text { system, or integrated technology }\end{array}$ \\
\hline \multirow{4}{*}{$\begin{array}{l}\text { Synopsizing } \\
\underline{\text { Concept- }} \\
\underline{\text { ualization }}\end{array}$} & Analysts & Designers \\
\hline & $\begin{array}{l}\text { Understand and improve the "leaf or } \\
\text { tree" }\end{array}$ & $\begin{array}{l}\text { Understand and improve part of or the } \\
\text { entire "forest or ecosystem" }\end{array}$ \\
\hline & $\begin{array}{l}\text { Inspired to produce "academic" } \\
\text { products such as validated theories, } \\
\text { reference-able results, and computer } \\
\text { simulations within the discipline }\end{array}$ & $\begin{array}{l}\text { Inspired to produce "design shop"-like } \\
\text { products such as creating breakthrough } \\
\text { system capabilities, innovations, or } \\
\text { integrated technologies }\end{array}$ \\
\hline & $\begin{array}{l}\text { Interdependency with the design- } \\
\text { oriented sub-culture: } \\
\text { - Guided by the needs of the "forest" } \\
\text {-Goals are pruned to be more } \\
\text { independent, such as focusing on type } \\
\text { of "leaf or tree in one type of } \\
\text { environment" }\end{array}$ & $\begin{array}{l}\text { Interdependency with the physics-oriented } \\
\text { sub-culture: } \\
\text { - Built upon a deep understanding of the } \\
\text { "leaves and trees" by themselves or others. } \\
\text { - Goals are nurtured to understand the } \\
\text { "forest's interdependencies in many } \\
\text { environments, such as decay to enable } \\
\text { growth in swamps, meadows, or a larger } \\
\text { ecosystem." }\end{array}$ \\
\hline
\end{tabular}

In general, the higher engineering goal of both approaches is the same, expressed by one MDO researcher with 30 years experience as: "motivated by improving the system or making something [operate], making it safer for people, making it better for the economy or making it [better for the environment]." The principal difference is approach towards this end. It is important to note that many single-discipline researchers also focus their work toward a more design-oriented approach and deeply enjoy cross-disciplinary research. 
Facilitating productive work that bridges the two foci noted in Table $\mathbf{2}$ requires attention to multiple facets including social, organizational, and cognitive aspsects as well as engineering aspects. As noted earlier, the data obtained strongly indicates that MDO's most influential function is in addressing the engineering aspects and providing information to support cognitive improvements such as increased understanding of other disciplines. Addressing the other aspects of cross-disciplinary work has many rewards and significant challenges. As noted in several research articles, working across discipline boundaries is the domain of constructive argument, increased learning, creativity, cooperation, and overcoming ignorance. ${ }^{39,40,42,45,54,55}$ Many respondents articulated the inherent tensions and a sense of messiness in their interactions. Klein describes that interdisciplinarity "requires accepting, from the outset, the unforeseeable and the productive role of misunderstanding. A sense of the new and surprising is decisive in mutual exchange and dialogue. The result is not necessarily consensus or unity; dissent will remain a thorny issue." 54 Accordingly, research on interdisciplinarity repeatedly emphasizes challenges of communications where "all interdisciplinary activities require translation and negotiation." 54 Interdisciplinarity is best described as knowledge integration where "the goal, purpose, or result of the research process is to construct a more comprehensive understanding." 34

In studying the challenges for distributed, interdisciplinary teams, Haythornthwaite et al, note that while much literature and organizational effort is focused on making "tacit knowledge explicit for transfer to others" their research suggests that "contemporary teams face a more complex set of issues as they engage in joint knowledge construction. Contemporary team members find that they cannot simply transfer their previous collaborative skills to a widely distributed, interdisciplinary arena, but must continually renegotiate a wide range of research and work practices thought to be already established." Their research also distinguishes novices and experts suggesting that while novices may focus on "transfer," experts on distributed, interdisciplinary teams focus on "joint problem-solving, shared cognition and co-construction of meaning." 40

Table 3 displays example responses from single-discipline researchers in working with MDO researchers to address unconventional technologies. In addition, Table 4 presents example responses from MDO researchers and one of many like-minded single discipline researchers.

Table 3 Four Single-Discipline Researchers from different disciplines, each with 20 or More Years Experience, Describing Working with MDO Researchers

- "We have a very nuanced multi-dimensional world and trying to take all this vast subtleties you understand and try to collapse into something that you can pass up and it can be used and not misrepresented at the system level. That's the pain and the cost to working that [MDO]."

- "They're looking at too high a level. I'm looking down at what's happening at [a significantly lower] level. They don't even care if I've got [A], [B], and [C], or if I've got [D]. So, from a [discipline X] world there's a huge difference in how you [work that technology] and how that actually performs. Because the systems level guys are looking at the [whole] system, they've simplified all of my problems down to-or all of my solutions down to a single problem to the point that it's oversimplified. So, they're not capable of giving me [trades on our technologies]."

- "I still don't know if that [MDAO] goes down and captures everything. You know with MDAO, you're kind of looking at a suite of tools that may be pre-existing, or existing and they may not fully capture all of the various disciplines... [such as] other technologies that they might be able to pull in and integrate to arrive at another solution. ... in terms of looking at what's out there and what's emerging, and integrating all the different technologies together, I think, is different from doing a [model-based systems] analysis."

- "If the systems analysis person jumps to a conclusion without having enough information-I think systems analysis people probably tend to have a fairly broad perspective, I think, because they've had to work on a lot of different stuff. It becomes tempting for that person, I think, to feel that I have a perspective and I can probably tell sooner than those specialists that they're going off on a track - it may be fine for them, but it's not going to fly. I think this may be tempting for a systems engineer to make-not quite snap judgments, but too early judgments on things." 
Table 4 Three MDO Researchers and One Single Discipline Researcher Describing Working with Single-Discipline Focused Researchers

Three Systems Analysts (with over 30 Years Experience) who largely do Early Conceptual Design Related Work:

- "[Working across disciplines] has to be not only just looked at favorably but it has to be, 'You haven't finished. Yes, that's all very interesting. That's all well and good. That was a great paper. You know, congratulations on getting accepted to the journal but how did you make our system ... better?'”

- 'The majority of them don't care whether what they're working on ever winds up [being used on a real system] or not. They're just happy to hunker down and do their thing. They get some sort of self-satisfaction of-here's my prejudice coming in-oh, they get to publish and go to this conference and that conference, and their colleagues all applauded when they were done. ... Most of the [managers] have come up through the ranks with the same sort of thinking. Our whole [organization] is sort of built around research and paper publishing, 'Oh, you got best paper for the year.' That's something. ... They don't value the same things that we value, that I value, in [my part of the] organization of having an impact on the world, on our product, on what we do. Maybe they do."

- "The old-style [way of doing things around here] says you do an experiment, you do the analysis, and they match and you put your little chart up there. Done. Well, a lot of this multidisciplinary stuff, doing the experiment to prove that the analysis is right is just really difficult or expensive or hard to pull together and so that's why when you present just the analysis part [of the multidisciplinary work] you get no respect. ...You can do a simple little [coupon-like] test and explain to your boss that, 'Well, we could make the whole [system]-if you wanted the whole [system],' but you don't. Whereas it's hard to come up with a [coupon-like] test that proves that our [cross-disciplinary] conceptual design is working."

One Single-Discipline Researcher (with over 35 Years Experience) Who Leads Large CrossDisciplinary Teams:

- "They [the team members] have to show... how [their work] is connected to the ... goals and why this is important to do, and if you succeed, does anybody care? Or is it just something that allows you to get a publication in a journal? It's good for you, but it doesn't do anything to meet the [organization's] goals of [system level improvements]? ...People somehow seem to think it's just a research lab. We're going to just do research because I can get a publication. Unfortunately, that's how they get promoted, so there's a catch-22. ... May be contributing very well there, but it never realizes itself into a real product if you will, or enabling future products. A lot of people just write papers to go to conferences or to get promoted. I wish we could find a different way to do that."

These example responses portray the differing viewpoints regarding working across disciplines. The frustrations expressed derive from many sources including but not limited to misunderstandings, competing values, different mental models, and local organizational culture and incentives.

The cognitive gap between the MDO researchers and the single-discipline researchers may be a significant source of the frustrations mentioned. While an MDO researcher likely has an advanced understanding of the system with limited understanding of the new technology, the single-discipline researcher likely has an advanced understanding of the new technology with limited understanding of the system - and, the incentive system often encourages the latter. Marrying these two areas of expertise requires, at a minimum, an interdisciplinary knowledge integration where both groups interactively and reciprocally update and modify their incoming understanding and change their theories and methods as necessary.

However, often respondents described a multidisciplinary (not interdisciplinary) integration scenario where different single-disciplinary researchers independently provide information to an MDO researcher who integrates the different inputs. In the scenarios observed, the integration conducted by the MDO researcher enables the MDO researcher to obtain an increased interdisciplinary understanding of the varying disciplines and the system. However, many of the single-disciplinary researchers that supply the MDO research group with information do not interact between themselves. Thus, while they might update their single-disciplinary models with new data from the MDO group

14

American Institute of Aeronautics and Astronautics 
such as new boundary conditions or system operating conditions, their single-disciplinary understanding, knowledge, theories, and methods may not be significantly modified.

\section{Discussion}

Many respondents, both MDO researchers and single-disciplinary researchers, emphasized the challenges noted above. These challenges create a communication and cognitive gulf between MDO researchers and the single-disciplinary researchers. In essence, the two parties are speaking different disciplinary languages, working at different fidelities, and have different understandings of the technologies and the systems in which they would be integrated.

The lack of reciprocity in the relationship between MDO researchers and single-disciplinary researchers adds to these frustrations. Nearly every MDO researcher expressed frustration in obtaining sufficient information from single-disciplinary researchers for conducting their analyses, while singledisciplinary researchers noted there were difficulties in working with MDO to address unconventional technologies. MDO researchers state that they are constrained by the codes, performance data, and support they obtain from single-disciplinary researchers.

With an incentive system focused toward individual achievements, many MDO researchers and single-disciplinary researchers note that there is insufficient incentive for single-disciplinary researchers to proactively work with the MDO researchers. While all of the MDO researchers and single disciplinary researchers were enthusiastic and even passionate about working with unconventional technologies, incentives, existing codes, and communication were often constraining. Despite the significant challenges with working in an interdisciplinary environment, nearly all respondents described significant learning opportunities that resulted from the interactions. In a sense, the ignorance that is inherent to interdisciplinarity becomes useful. A senior researcher with extensive experience in interdisciplinary teams states: "It can't be codified.... The questions that [different disciplines] ask may be partially out of their ignorance, but that ignorance can also be brilliance because it asks a question." Another respondent with similar experience replies: "coming at problems from totally different directions seeing it from a new light can spawn new ideas." However to effectively exploit the diversity of thought in the interdisciplinary interactions, significant social capabilities are necessary as argument and personality differences are intrinsic to working across disciplines. In addition, the characteristic re-evaluations of roles, procedures, and existing knowledge are latent in the interactions and were difficult for many respondents though they enjoyed the intellectual advantages of cross-disciplinary work previously described.

The interdisciplinary interactive arena of $\mathrm{R} \& \mathrm{D}$ and early design for large systems is one where boundaries are not ignored, they are re-evaluated. Disciplinary knowledge is not erased, rather: "The worldview or perspective embedded in each disciplinary piece is extracted, compared, and evaluated for relevance. When conflicts are detected, they are clarified. They do not disappear, however, in a false unity that denies difference (Klein 1995; Klein and Newell 1996)." ${ }^{54}$ Many respondents, including managers and leaders, appeared to desire a more unified approach to interdisciplinarity and often tried to proactively dampen differences. Klein writes of this historical interdisciplinary ideal:

"The older interdisciplinary ideal was a world in which differences were to be overcome.

The reality is that differences matter. Even if negotiated and mediated, differences do not go away - they continue to create 'noise.' Misunderstandings, animosities, and competitions cannot be mitigated or glossed over. They must be taken seriously as attempts are made to spell out differences and their possible consequences. Interdisciplinarity conceived as communicative action does not trust that everything will work out if everyone will just sit down and talk to each other. Decades of scuttled projects and program belie the naïve faith that status hierarchies and hidden agendas will not interfere or that the individual with the greatest clout or loudest voice will not attempt to dominate. ${ }^{, 54}$

Klein's discussion highlights the inherently organizational, social, and cognitive nature of interdisciplinarity. Respondents were profuse in their comments regarding the social and interpersonal needs of interdisciplinarity in their organization. Every respondent noted that interpersonal aspects were central to working with other disciplines. Their descriptions of interpersonal topics were woven through all of the interviews. Ethnographic observations confirmed this. And literature on collective 
mind in organizations and interdisciplinarity also assert the significance of ongoing positive interpersonal relations.

In conceptualizing the collective mind, Weick notes there is "little room for heroic, autonomous individuals. A well-developed organization mind, capable of reliable performance is thoroughly social. It is built of ongoing interrelating and dense interrelations. Thus, interpersonal skills are not a luxury in high-reliability systems. They are a necessity." distributed organizing, Orlikowski describes "knowing is an ongoing social accomplishment, constituted and reconstituted in everyday practice." ${ }^{56}$ In researching "creative collectives" Hargadon and Bechky describe "mindful interactions across individuals" to provide for "a collective mechanism for generating solutions. ${ }^{, 57}$ Literature on interdisciplinarity is also consistent in stressing the criticality of social interactions. ${ }^{34,40,42,45,54}$

In this study, many respondents described the challenges previously mentioned and the importance of leaders who could mitigate them. The leaders that successfully fostered or enabled interdisciplinary interactions among researchers typically were not supervisory line managers or program managers nor MDO researchers, but rather were senior researchers, usually within a single discipline with exceptional interpersonal skills who often worked at a level lower than line managers or program managers. All respondents were unequivocal in stating there were specific individuals in their organizations who were exceptional at facilitating interdisciplinary interactions:

- "Some people are good facilitators without necessarily being aware of it."

- "There are some people who are good at seeing connections, who are good at seeing how working together is going to benefit the group and then also conveying that to people. ... There are people who are good at seeing it, but lack the people skills to bring the group on board."

The last respondent above sums up the comments of many and points toward a common misconception in management and leadership where it is assumed that those with the cognitive ability and professional training to understand system-level trades are the default focal point for crossdisciplinary interactions in their organizations, eventhough these individuals may not have the social skills to facilitate and sustain effective interpersonal interactions. Hence, both cross-discipline and social competence is required for enabling cross-disciplinary interactions. An MDO researcher with over 10 years experience describes his or her perspective:

"I think the most important part of MDAO is really the interpersonal part. I think we'veas a discipline-because I consider myself an MDAO researcher-I think we've got a handle on or we're moving toward getting a handle on the technical aspects of it. I mean there's always more research to be done but we understand very well about systems and optimization and configuration and things like that. Computational costs still a challenge but we're working on it. But we haven't really started to address the inter-personal issues. I think that's the most important."

Many respondents also explained that the organizational structure of cross-disciplinarity runs orthogonal to but not against the existing hierarchy. Disciplines interacted across buildings, line organizations, programs, and geographic regions via ad hoc personal connections throughout their large organizations in addition to working within formally structured cross-discipline teams. In the vignette below, one single-disciplinary researcher with over 25 years experience (who was wellrespected by their peers at being exceptional at leading interdisciplinary teams) describes an expanding and informal network that he or she hopes to enable through working across disciplines.

"Right now the 'between discipline' interaction has been much more personal connections, much more ad hoc, out of necessity. I need to [X]. So, tell me who out here [does that]. So, the [two] come together, not necessarily going through the MDAO person [or an official manager]. It's who do you know. It's that personal network in a lot of cases, I think. So, part of what I've done is build a personal network where everybody is introduced to each other. So, in forming this group, now we've got this personal network and my hope is that each one of these people goes back into their home organization, their stovepipe, but if somebody else says 'hey, I need an [X]'-and I've seen this happen - 'I've got an [X] question.' It's like, 'oh, well here's my [X] contact. So, all of a sudden now somebody else in one stovepipe is calling this $[X]$ contact in another stovepipe because of that personal connection. So, it doesn't go through the MDAO folks [or management] at all. After there gets to be a relationship and a trust that seems to happen more ad hoc.

16

American Institute of Aeronautics and Astronautics 
So, instead of having a centralized network, it becomes a distributed network where those connections happen discipline to discipline through the people that they know. So, each of those people [on our team] almost becomes a node on the map. So they've got all these other [X] people [as contacts], and this [team member] has all these other [Y] people [as contacts], and this [team member] has all these other [Z] people [as contacts]. So, this guy comes to [a team member] and [our team member] says, 'Oh, go talk to him [one of his contacts].' Then [that contact] says, 'Oh, go talk to [someone else that can help also],' as opposed to coming back up through the center[team leader]."

An important aspect of the findings is the significant influence of social capital on interdisciplinary interactions. Simply, positive social interrelations encouraged interdisciplinarity and negative interrelations discouraged it - regardless of the engineering need or management direction. Positive social interrelations are pivotal for addressing some of the negative emotional arousal associated with interdisciplinary interactions in large, dispersed engineering organizations. Negative emotional arousal stems from a variety of sources including: ignorance; confusion; new people; new organizations and related culture; potential career impacts; and, ego. Ignorance resulted from a lack of understanding of other disciplines. Confusion resulted from unfamiliar processes and ambiguity. New people, organizations, and culture created discomfort and considerable mitigated speech and slowed action. A single-discipline focused incentive system often resulted in respondents being concerned about the potential career impacts of working across disciplines and, all of these challenges impact ego.

Mitigating these challenges requires continual attention to building positive social capital. The tenets of social capital ${ }^{49,58,59}$ coincide well with what respondents described as needed elements of interacting across disciplines. Their comments centered on the need for respectful engagement and valuing people's contribution to the interdisciplinary interaction. Respondents also described a need for trust - both intellectual and social trust. Intellectually, respondents wanted their ideas to be heard and their ignorance not to be a source of embarrassment. Socially, respondents wanted to be welcomed and valued as colleagues or friends.

A need for generalized reciprocity was also clear and consistent. When interdisciplinarity tended toward a one-way exchange, respondents were reluctant to engage fully and were often dissatisfied with the interaction. Reciprocity and joviality are inherent in the most effective interdisciplinary interactions. All of these aspects - respect, trust, reciprocity, and joviality - are also key tenets of positive social capital. ${ }^{5,50}$ The literature on positive social capital also notes that there are significant, organization-wide benefits to building positive social capital that improve interdisciplinary interactions as well the broader organization. These include: broader thinking, better learning, enhanced cooperation, greater attachment of employees, increased job satisfaction, and other benefits., 50

While social, organizational, and engineering are significant aspects of interdisciplinary interactions in R\&D and early design of LaCES, the heart of the interactions may best be described as intellectual transformation. Thinking, understanding, awareness, and knowledge evolve in the interactions where a complementary and interdependent relationship between single disciplinarity and interdisciplinarity was observed. Discipline understanding is foundational for interdisciplinarity. And interdisciplinarity enriches single disciplinarity by spawning new ideas, updating thinking, increasing system relevance, and exploiting new single disciplinary findings. While the two approaches may be accomplished in absence of the each other, the richness, effectiveness, and efficiency of both single disciplinary and interdisciplinary research is greatly improved when the two work in concert.

Particularly for LaCES design, disciplinary depth is requisite for understanding complicated phenomena and interdisciplinary understanding is requisite for addressing the interdependencies that are intrinsic to the system. In short, LaCES cannot be understood nor effectively designed without both single-disciplinary depth and interdisciplinary breadth. However, this is not an argument that may be simplified to a need for specialists and generalists. Rather, the data clearly indicate that interdisciplinarity is practiced in a wide range of time investment from only $5 \%$ of a respondent's effort to $95 \%$ of his or her effort with this effort varying as needed for different projects.

Another aspect of interdisciplinary intellectual transformation that is particularly relevant for LaCES is the discovery of the unknown between conventional knowledge domains, mathematical models, system elements, organizational structures, etc. The unknowns may be new technological capabilities or potentially dangerous couplings that may only be discovered through human-to-human interdisciplinary interactions. For complex systems, often problems and opportunities do not arise where you are looking but rather where you are not looking. ${ }^{60}$ More comprehensive system knowledge 
is interactively constructed through the interdisciplinary discussions that include both debate and affirmation. Opportunities for creativity are created and greater awareness of system interdependencies is increased.

\section{Conclusion}

We presented findings from a larger study on interactions among researchers and engineers from different disciplines working on large complex systems. We focused our discussions on findings that pertain to MDO practice and human interactive practices among the disciplines and groups in the large, dispersed organizations that deign these systems. The findings show that cognitive, social, and organizational aspects are the primary drivers of cross-disciplianry interactions. While MDO continues to serve a critical role in cross-disciplinary research and it is essential for enabling LaCES design, it is one of several important enablers for effective cross-disciplinary research, development and design of large complex systems. Just implementing MDO strategies does not meet all the challenges presented by such systems.

\section{Acknowledgments}

The authors gratefully acknowledge the invaluable contributions of Drs. Colleen Seifert and Shanna Daly from the University of Michigan.

\section{References}

${ }^{1}$ Cumming, M. "Flexible and distributed coordination models for collaborative design," 2002.

${ }^{2}$ Weick, K. E., and Roberts, K. H. "Collective Mind in Organizations: Heedful Interrelating on Flight Decks," Administrative Science Quarterly Vol. 38, No. 3, 1993, p. 357. doi: $10.2307 / 2393372$

${ }^{3}$ Wasserman, S., and Faust, K. Social network analysis: Methods and applications: Cambridge university press, 1994.

${ }^{4}$ Weick, K. E., Sutcliffe, K. M., and Obstfeld, D. "Organizing for high reliability: Processes of collective mindfulness," Crisis Management Vol. 3, 2008, pp. 81-123.

${ }^{5}$ Baker, W., and Dutton, J. E. "Enabling Positive Social Capital in Organizations," Exploring Positive Relationships at Work: Building a Theoretical and Research Foundation. Lawrence Erlbaum Publishers, Mahwah, NJ, 2007.

${ }^{6}$ Simpson, T. W., and Martins, J. R. "Multidisciplinary design optimization for complex engineered systems: report from a national science foundation workshop," Journal of Mechanical Design Vol. 133, No. 10, 2011, p. 101002.

${ }^{7}$ Skyttner, L. General systems theory: ideas \& applications: World Scientific, 2001.

${ }^{8}$ Systems, I. W. G. o. E. C. "InterAgency Working Group Position Paper." Vol. Feb 2014, 2014.

${ }^{9}$ Ben-Ari, G. Organizing for a complex world: developing tomorrow's defense and net-centric systems: Center for Strategic \& International Studies, 2009.

${ }^{10}$ Mitchell, M. Complexity: A guided tour: Oxford University Press, USA, 2009.

${ }^{11}$ Bar-Yam, Y., Ramalingam, C., Burlingame, L., and Ogata, C. Making things work: solving complex problems in a complex world: NECSI, Knowledge Press, 2004.

${ }^{12}$ Minai, A. A., Braha, D., and Bar-Yam, Y. "Complex engineered systems: A new paradigm," Complex engineered systems. Springer, 2006, pp. 1-21.

${ }^{13}$ Bloebaum, C. L., and McGowan, A.-M. R. "The Design of Large-Scale Complex Engineered Systems: Present Challenges and Future Promise," 12th AIAA ATIO Conference and 14th AIAA/ISSMO MA\&O Conference. Vol. AIAA Paper 2012-5571, AIAA Paper number: 2012-5571, Indianapolis, IN, AIAA Paper 2012-5571, 2012.

${ }^{14}$ Pratt, M. G. "For the Lack of a Boilerplate: Tips on Writing Up (and Reviewing) Qualitative Research," Academy of Management Journal Vol. 52, No. 5, 2009, pp. 856-862.

${ }^{15}$ Jones, P. H., Chisalita, C., and Veer, G. C. v. d. "Cognition, technology, and work: special issue on collaboration in context: cognitive and organizational artefacts," Cognition, Technology \& Work Vol. 7, No. 2, 2005, pp. 70-75.

${ }^{16}$ Leydens, J. A., Moskal, B. M., and Pavelich, M. J. "Qualitative methods used in the assessment of engineering education," Journal of Engineering Education-Washington Vol. 93, No. 1, 2004, pp. 65-72.

${ }^{17}$ Daly, S., McGowan, A., and Papalambros, P. "Using qualitative research methods in engineering design research," DS 75-2: Proceedings of the 19th International Conference on Engineering Design (ICED13), Design for Harmonies, Vol. 2: Design Theory and Research Methodology, Seoul, Korea, 19-22.08. 2013. 2013.

${ }^{18}$ Maxwell, J. A. "Designing a qualitative study," Handbook of applied social research methods, 1998, pp. 69-100. 
${ }^{19}$ Kvale, S., and Brinkmann, S. Interviews: Learning the craft of qualitative research interviewing: Sage Publications, Inc, 2008.

${ }^{20}$ Chilcott, J. H. "Where Are You Coming From And Where Are You Going - The Reporting Of Ethnographic Research," American Educational Research Journal Vol. 24, No. 2, 1987, pp. 199-218.

doi: 10.3102/00028312024002199

${ }^{21}$ Emerson, R. M., Fretz, R. I., and Shaw, L. L. Writing ethnographic fieldnotes: University of Chicago Press, 2011.

${ }^{22}$ Humphreys, M., and Watson, T. J. "Ethnographic practices: from "writing-up ethnographic research" to "writing ethnography"," Organizational Ethnography: Studying the Complexities of Everyday Organizational Life. London: Sage, 2009, pp. 40-55.

${ }^{23}$ Alvesson, M. "Methodology for close up studies - struggling with closeness and closure," Higher Education Vol. 46, No. 2, 2003, pp. 167-193.

doi: 10.1023/a:1024716513774

${ }^{24}$ Edwards, B. "Deep insider research," Qualitative Research Journal Vol. 2, No. 1, 2002, pp. 71-84.

${ }^{25}$ Brannick, T., and Coghlan, D. "In defense of being - "Native" the case for insider academic research," Organizational Research Methods Vol. 10, No. 1, 2007, pp. 59-74.

doi: $10.1177 / 1094428106289253$

${ }^{26}$ Lofland, J., and Lofland, L. H. Analyzing social settings: a guide to qualitative observation and analysis. Belmont, Calif.: Wadsworth, 1995.

${ }^{27}$ Boeije, H. "A purposeful approach to the constant comparative method in the analysis of qualitative interviews," Quality \& Quantity Vol. 36, No. 4, 2002, pp. 391-409.

doi: 10.1023/a:1020909529486

${ }^{28}$ Marshall, C., and Rossman, G. B. Designing qualitative research: Sage Publications, Inc, 2010.

${ }^{29}$ Ryan, G. B., H. Russel. "Techniques to Identify Themes," Field Methods Vol. 15, No. No. 1, 2003, pp. 85-109. doi: $10.1177 / 1525822 \mathrm{X} 02239569$

${ }^{30}$ Strauss, A., and Corbin, J. Basics of qualitative research: Grounded Theory Procedures and Techniques. Newbury Park, CA: Sage Publications, Inc., 1990.

${ }^{31}$ Gioia, D. A., and Chittipeddi, K. "Sensemaking And Sensegiving In Strategic Change Initiation," Strategic Management Journal Vol. 12, No. 6, 1991, pp. 433-448.

doi: $10.1002 / \mathrm{smj} .4250120604$

${ }^{32}$ Case, J. M., and Light, G. "Emerging methodologies in engineering education research," Journal of Engineering Education Vol. 100, No. 1, 2011, pp. 186-210.

${ }^{33}$ Porter, A., and Rafols, I. "Is science becoming more interdisciplinary? Measuring and mapping six research fields over time," Scientometrics Vol. 81, No. 3, 2009, pp. 719-745.

doi: 10.1007/s11192-008-2197-2

${ }^{34}$ Repko, A. F. Interdisciplinary Research Process and Theory, Second Edition: Sage, 2012.

${ }^{35}$ Rhoten, D. "Interdisciplinary research: Trend or transition," Items and Issues Vol. 5, No. 1-2, 2004, pp. 6-11.

${ }^{36}$ Roberts, J. A., and Barnhill, R. E. "Engineering togetherness (an incentive system for interdisciplinary research)," Frontiers in Education Conference, 2001. 31st Annual. Vol. 2, IEEE, 2001, pp. F2G-23-7 vol. 2.

${ }^{37}$ Robertson, D. W., Martin, D. K., and Singer, P. A. "Interdisciplinary research: putting the methods under the microscope," BMC Medical Research Methodology Vol. 3, No. 1, 2003, p. 20.

${ }^{38}$ Wear, D. N. "Challenges to interdisciplinary discourse," Ecosystems Vol. 2, No. 4, 1999, pp. 299-301.

${ }^{39}$ Gooch, J. C. "The dynamics and challenges of interdisciplinary collaboration: a case study of," Professional Communication, IEEE Transactions on Vol. 48, No. 2, 2005, pp. 177-190.

${ }^{40}$ Haythornthwaite, C., Lunsford, K. J., Bowker, G. C., and Bruce, B. C. "Challenges for research and practice in distributed, interdisciplinary collaboration," New infrastructures for science knowledge production, 2006, pp. 143166.

${ }^{41}$ Karanika-Murray, M., and Wiesemes, R. Exploring avenues to interdisciplinary research: from cross-to multi-to interdisciplinarity: Nottingham Univ Pr, 2009.

${ }^{42}$ Klein, J. T. "Evaluation of interdisciplinary and transdisciplinary research: a literature review," American journal of preventive medicine Vol. 35, No. 2, 2008, pp. S116-S123.

${ }^{43}$ Andreasen, N. C., and Brown, T. "Facilitating interdisciplinary research," National Academy of Science National Academy Engineering and the Institute of Medicine, 2005.

${ }^{44}$ Augsburg, T. Becoming interdisciplinary: An introduction to interdisciplinary studies: Kendall/Hunt Pub., 2006.

${ }^{45}$ Frodeman, R., Klein, J. T., and Mitcham, C. The Oxford handbook of interdisciplinarity: Oxford University Press Oxford, 2010.

${ }^{46}$ Merriam-Webster. "Webster's Ninth New Collegiate Dictionary." Springfield, MA, 1991.

${ }^{47}$ Dutton, J. E., and Ragins, B. R. Exploring positive relationships at work: Building a theoretical and research foundation: Lawrence Erlbaum Associates, 2007.

${ }^{48}$ McGowan, A.-M. R. "Interdisciplinary Interactions During R\&D and Early Design of Large Engineered Systems," Design Science. Vol. PhD, University of Michigan, College of Engineering, NASA Langley, 2014, p. 198. 
${ }^{49}$ Baker, W. E. Achieving Success through Social Capital: Tapping the Hidden Resources in Your Personal and Business Networks. University of Michigan Business School Management Series: San Francisco: Jossey-Bass, 2000 .

${ }^{50}$ Dutton, J. "Energize your workplace: How to build and sustain high-quality relationships at work." San Francisco, CA: Jossey-Bass, 2003.

${ }^{51}$ Barney, J. B. "Organizational Culture: Can It Be a Source of Sustained Competitive Advantage?," The Academy of Management Review Vol. 11, No. 3, 1986, pp. 656-665. doi: $10.2307 / 258317$

${ }^{52}$ O'Reilly, C. A., Chatman, J., and Caldwell, D. F. "People and organizational culture: A profile comparison approach to assessing person-organization fit," Academy of Management Journal Vol. 34, No. 3, 1991, pp. 487516.

${ }^{53}$ Kotter, J. P. Corporate culture and performance: SimonandSchuster. com, 2008.

${ }^{54}$ Klein, J. T. Crossing boundaries: Knowledge, disciplinarities, and interdisciplinarities: University of Virginia Press, 1996.

${ }^{55}$ Olsen, P. B., and Heaton, L. "Knowing through design," Design Research: Synergies from Interdisciplinary Perspectives. Routledge, 2010, p. 79.

${ }^{56}$ Orlikowski, W. J. "Knowing in practice: Enacting a collective capability in distributed organizing," Organization Science Vol. 13, No. 3, 2002, pp. 249-273.

${ }^{57}$ Hargadon, A. B., and Bechky, B. A. "When collections of creatives become creative collectives: A field study of problem solving at work," Organization Science Vol. 17, No. 4, 2006, pp. 484-500.

${ }^{58}$ Baker, W., Cross, R., and Wooten, M. "Positive organizational network analysis and energizing relationships," Positive organizational scholarship: Foundations of a new discipline, 2003, pp. 328-342.

${ }^{59}$ Baker, W. E. Networking smart: how to build relationships for personal and organizational success: iUniverse, Incorporated, 2000.

${ }^{60}$ Jones, K. H., Parker, P. A., Detweiler, K. N., McGowan, A.-M. R., Dress, D. A., and Kimmel, W. M. "Analysis and Perspectives from the Complex Aerospace Systems Exchange (CASE) 2013." 